\title{
Tlacauhtli, altepetl y tlalli: Conceptos básicos de estructuración del espacio, territorio y tierra en el México pre-colombino
}

\author{
Daniel Astorga Poblete ${ }^{1}$ \\ Recibido: 15 de septiembre de 2014 - Aceptado: 15 de octubre de 2014
}

\begin{abstract}
Resumen
La idea de "espacio" dentro del mundo nahua precolombino se organiza bajo tres ideas: tlacahuitli, altepet/ y tlalli. Cada concepto se analiza dentro de la cosmovisión e historia de esta cultura. En este artículo se sostiene que la estructuración de estos tres conceptos viene dada por una idea en común en base al movimiento, lo cual explica la interrelación entre las tres dimensiones del espacio nahua.
\end{abstract}

Palabras clave: Espacio nahua, territorialidad, distribución de tierras, espacio cosmológico

\section{Tlacauhtli, Altepetl y Tlalli: Basic concepts on space and land structure in pre-columbian Mexico}

\begin{abstract}
The idea of "space" in the pre-Columbian Nahua world is organized under three concepts: tlacahuitli, altepetl and tlalli. Each concept is analyzed within the worldview and history of this culture. This article argues that the structure of these three concepts is given by a common idea based on the movement, which explains the interrelationship among the three dimensions of Nahua space.
\end{abstract}

Keywords: Nahua space, territoriality, land distribution, cosmological space

Chileno. Ph. D. (c), Romance Studies, Duke University, EE. UU. Académico de la Universidad Finis Terrae. E-mail: dea12@duke.edu. 


\section{Tlacauhtli, Altepetl e Tlalli: Conceito básico da estruturação do espaço, território e terra no México pré-colombino

\begin{abstract}
Resumo
A idéia de "espaço" dentro do mundo nahua pré-colombino é organizado em três ideias: tlacahuitli, altepetl e tlalli. Cada conceito é analisado dentro da cosmologia e a história desta cultura. Este artigo argumenta que a estruturação destes três conceitos decorre de uma ideia em comum com base em movimento, o que explica a inter-relação entre as três dimensões do espaço nahua.
\end{abstract}

Palavras-chave: espaço nahua, territorialidade, distribuição de terras, espaço cosmológico.

El siguiente trabajo intenta poner en relación tres conceptos esenciales dentro de la organización espacial del valle central de México previo a la llegada de los españoles: tlacauhtli, correspondiente a "espacio"; altepetl, similar a la noción de "estado étnico dominador de un territorio"; y tlalli, que significa "tierra". A pesar de que estos tres conceptos pertenecen a tres niveles distintos de organización de una realidad espacial, se demuestra que ellos se estructuran de una manera similar. Utilizando los relatos ancestrales de la creación del mundo mesoamericano y la historia social y económica de la Triple Alianza de México-Tenochtitlan, Texcoco y Tlacopan, se observa que la ordenación de estos tres niveles de espacialidad se establece de acuerdo a un patrón común regido por un concepto: movimiento. De esta manera, se da una consistencia, coherencia e integración a estos distintos ámbitos de la organización del mundo y la tierra.

\section{Tlacauhtli y la construcción del espacio cosmológico}

Nuestro primer concepto dentro de la lengua de los nahuas del valle central de México será tlacauhtli, el cual se refiere a la idea de espacio en su sentido general. En su Vocabulario en Lengua Castellana y Mexicana, Fray Alonso de Molina define tlacauhtli como "espacio de lugar, o cosa dejada, o sobras". Al respecto, Miguel León Portilla ha esbozado una teoría con respecto al concepto. Para él, tlacauhtli tendría una estrecha relación con el vocablo cahuitl, que corresponde a la palabra "tiempo" en español. Ambos vocablos en náhuatl estarían derivados del verbo cahua, que vendría a significar "dejar algo o llevar alguna cosa a otra parte". Por lo tanto, según León-Portilla, "El denominador de ambos conceptos es 'dejar', 'ir dejando', cuando se refiere al tiempo y 'haber sido ya dejado permanentemente', cuando se refiere al espacio" (2003: 181). La mejor manera de entender esta idea de que el espacio es esta "cosa dejada", es observando la primera página del códice Fejérváry-Mayer. (Figura 1) 


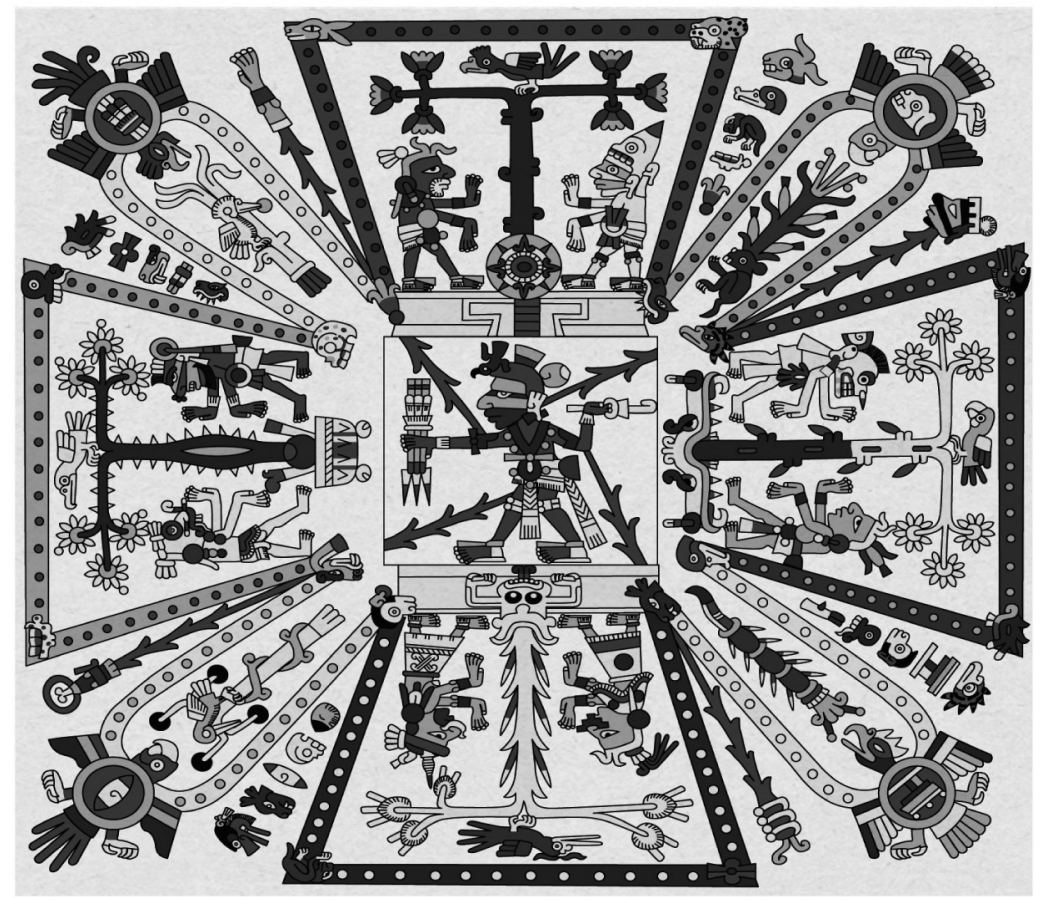

Figura 1 Primera página del Códice Fejérváry-Mayer

Lo que nos muestra esta imagen es una expresión de la organización del tiempo y del espacio. Por un lado, tlacauhtli se organiza en cinco ejes distribuidos en cuatro direcciones y un centro. Comenzando desde arriba y en sentido contrario a las manecillas del reloj, tenemos Tonatiuh ichan, "la casa del sol". En esta dirección, que correspondería al Oriente, tenemos un árbol de color azul de hermosas flores (xiloxochitl) con un quetzal, y un altar con el disco solar. A cada lado tenemos a Iztli, el Cuchillo del Fuego, y a Tonatiuh. En la dirección correspondiente al Norte, lo marca un árbol llamado "pochote espinoso" de color azul, con un halcón, una vasija o un brasero con punzones y una pelota de hule, y los dioses Tlaloc, el Terrestre, dios de la lluvia; y Tepeyollotl, Corazón de la Montaña, Dueño de los montes y de los animales que viven allí. Luego, tenemos Cihuatlampa, "el lado de las mujeres", correspondiente al Poniente, marcado por un árbol llamado huizache con un colibrí, una plataforma con un animal, una especie de insecto o ser espectral que carga la luna, y dos diosas: Chalchiuhtlicue, Falda de Jade, Diosa del agua de los ríos y lagunas; y Tlazolteotl, Diosa del Tejer y de la Sexualidad. Por último, tenemos la dirección Sur a la que los nahuas llamaban Mictlampa, pero que los Aztecas referían al Norte como "el rumbo del reino de los muertos". Aquí podemos observar un árbol de cacao con un papagayo, y a los dioses Cinteotl, Dios del Maíz, y Mictlantecuhtli, Señor 
del Reino de los Muertos. En el centro de esta cuatripartición tenemos a Xiuhtecuhtli, Señor del Fuego; y dividido en cuatro partes (cabeza, mano, pierna y costillas), a Tezcatlipoca.

Junto con la organización del espacio cosmológico en cuatro divisiones más un centro, tenemos la estructuración del tiempo llamado tonalpohualli o calendario. Los aztecas usaban un calendario sagrado consistente en veinte trecenas ( $20 \times 13=260$ días), lo que marcaba un año. Si observamos los trapezoides que encierran a los dioses de cada orientación cardinal y los lazos que los unen, encontraremos un camino marcado por 12 días más uno de los veinte signos. Los aztecas contaban los días de 1 a 13, con un signo entre los veinte diversos. Si comenzásemos a leer desde el día 1 Cipactli (Lagarto), que se encuentra en el extremo inferior derecho del cuadrante del Oriente, tendríamos que seguir la cuenta 2 Ehecatl, 3 Calli, 4 CuetzpaIlin, hasta llegar a 13 Acatl, en donde comenzaría una nueva trecena con 1 Ocelotl. Esto nos daría una estructura de cinco signos para cada rumbo espacial conformados por los cuatro signos del trapezoide más un quinto signo del lazo a su izquierda. Ahora bien, al lado de cada lazo observamos cinco signos que corresponderían a otra manera de organizar espacialmente las trecenas. Si dividimos la cuenta de las trecenas en cuatro bloques, y superponemos esos bloques, nos quedará la misma estructura de cuenta de los días que aparece en el Códice Borgia. Como explican Ferdinand Anders, Maarten Jansen y Gabina Pérez: "Ahora se hace una división más compleja y abstracta, que tiene como resultado otro sistema de orientar las trecenas. Obsérvese cómo cambia la orientación en los dos sistemas: solamente dos trecenas de las cinco de cada grupo reciben la misma orientación. La ambigüedad que resulta es propia del arte adivinatorio" (1994: 173). Por último, una tercera estructura temporal en el códice vendría dada por la orientación de los años que se enmarcaba en un ciclo de 365 días o año solar que los nahuas estructuraban en el xiuhpohualli. Si se observan los lazos ovalados que conectan los trapezoides, arriba de aquellos hay cuatro escudos rojos con bordes amarillos que llevan un signo cada uno. A estos signos se les llama "portadores de los años" y se ocupaban para nombrar los años en el xiuhpohualli o calendario solar. Estos signos son Acatl (Caña), Tecpatl (Cuchillo), Calli (Casa) y Tochtli (Conejo). Para entender por qué son sólo estos signos los que determinan los años, debemos considerar que los aztecas concebían el año solar en 365 días que estaban estructurados en diez y ocho trecenas $(18 \times 13=360)$ más cinco días aciagos también llamados nemotemi. Si comenzamos a contar desde 1 Acatl en forma continua hasta 365, el primer día del nuevo año será 2 Tecpatl, luego el siguiente año 3 Calli, 4 Tochtli, 5 Acatl, etc. Explicado matemáticamente, 365/13=28 y nos sobra 1, lo que hace avanzar el número del siguiente portador con una unidad; y $365 / 20=18$ y sobran 5 , lo que hace avanzar el signo de este en cinco posiciones. 
Una vez entendida la primera página del Códice Fejérváry-Mayer, se hace más fácil comprender la relación entre cahuilt y tlacauhtli, tiempo y espacio. Si la idea subyacente en ambos conceptos es la de "dejar algo" en un espacio o tiempo, podemos pensar que "lo que va siendo dejado" en el calendario es el tiempo que acontece en el espacio. Tal manera de experimentar esta unión no se hace tan difícil de concebir si es que nos olvidamos de toda manera de medir el tiempo por medio de artefactos modernos como los relojes y observamos el movimiento del sol para determinar el tiempo. En ese instante, tanto espacio como tiempo entran en una complicidad tal que sería difícil poder conceptualizarlos de una manera diferente. Por lo tanto, tlacauhtli vendría a conformar una idea de espacio cosmológico en constante relación con el movimiento del sol y su circulación espacio-temporal. Para entender esta relación, sería necesario volver a la narración de los cinco soles, la cual explicaba la creación del universo y el mundo.

Para los antiguos nahuas, el mundo ha tenido cuatro épocas cósmicas y, actualmente, vivimos en una quinta. Cada una de estas épocas ha sufrido un cataclismo que ha terminado con el mundo, sus habitantes han muerto o se han visto transformados en animales $u$ otros seres. A cada época le ha correspondido un Sol en particular que ha iluminado el mundo. A la primera época le correspondió Nahui Ocelotl (4 Jaguar); a la segunda Nahui Ehecatl (4 Viento); a la tercera, Nahui Quiyahuitl (4 Lluvia de Fuego); y, a la cuarta, Nahui Atl (4 Agua). Hoy en día viviríamos en el Sol llamado Nahui Ollin (Sol de Movimiento). Tal como lo señala Wayne Elzey, la sucesión de estas épocas y soles no está determinada por un desarrollo o evolución, sino que son Soles que terminan su reinado abruptamente, aniquilando casi todo lo que ha sido creado hasta el momento (Elzey, 1976: 123). Ahora bien, es el Quinto Sol el que marca un distanciamiento con los demás de una manera más radical. Para que el Quinto Sol existiese, los cuatro dioses, quienes habían dominado el mundo a la manera de soles, debían reunirse para equilibrar sus fuerzas y dar creación al nuevo Sol. De esta manera, Tlatlauhqui Tezcatlipoca, Yayauhqui Tezcatlipoca, Quetzalcoatl y Huitzilopochtli se reúnen en Teotihuacán y dan origen al Sol gracias al sacrificio de un pequeño dios llamado Nanahuatzin, quien se sacrifica a la hoguera y se transforma en el Sol. Finalmente, debido a que este Sol no se movía, los cuatro dioses decidieron hacer un sacrificio de sangre para que el Sol se moviese.

De la historia de los Cinco Soles podemos sacar dos conclusiones. Por un lado, para la existencia de la quinta edad y Quinto Sol es necesario que los cuatro soles anteriores, en otras palabras, los cuatro dioses que habían iluminado el mundo, pudiesen armonizar en un punto y equilibrar sus fuerzas para crear la quinta edad. En este sentido, el mundo comienza en el momento en que aparece el Quinto Sol y, por ende, este pueda comenzar a 
recorrer la tierra para alumbrar su faz. Esto quiere decir que la existencia del mundo, en cuanto a espacio, sólo se concibe cuando existe el movimiento del Sol. En otras palabras, para que exista el mundo, cahuitl y tlacauhtli, o el complejo tiempo/espacio, debe existir ollin (movimiento). Por otro lado, lo que permite que exista ollin (movimiento) es la conjunción armoniosa y equilibrada entre los cuatro dioses.

Cada uno lucha y gobierna un segmento del espacio y del tiempo, y esa lucha crea un equilibrio o quinto punto que se refiere al Quinto Sol, pero también un centro que genera una estabilidad y caos (ollin y nepant/a). Por lo tanto, lo que estructuraría al concepto de tlacauhtli (y también cahuitl) o, mejor dicho, lo que organiza el mundo cosmológico, es la interacción de dos elementos cruciales: la armoniosa conjunción de los cuatro puntos, cuatro dioses o cuatro soles y la creación de este centro que es el Quinto Sol; y el movimiento que permite la alternación de estos cuatro dioses para que gobiernen el mundo y mantengan el Quinto Sol². Por último, veamos la historia de Cipactli, una especie de caimán, por medio del cual los dioses hicieron la tierra, para corroborar la idea de la división cuatripartita del mundo y la interrelación armoniosa de estas partes para la subsistencia del mundo.

En un comienzo, antes de que existieran los dioses como Quetzalcoatl y Huitzilopochtli, existía un solo dios llamado Ometeotl, el cual se subdividía en una parte masculina y otra femenina: Ometecuhtli y Omecihuatl, respectivamente. Como explica Jacques Soustelle: "Ometecuhtli es el dios protector del primer signo del calendario, Cipactli, el monstruo mítico que lleva la Tierra sobre su espalda. Omecihuatl es la patrona del último signo, Xochitl, la flor..." (Soustelle, 1996: 99). Este ser primigenio era sustancia, la cual se dividió en dos partes opuestas y complementarias: la tierra y el cielo. De esta división se liberaron sustancias divinas, las que crearon a los cuatro dioses: Tlatlauhqui Tezcatlipoca, Yayauhqui Tezcatlipoca, Quetzalcoatl y Huitzilopochtli. Al mismo tiempo, como explica Alfredo López-Austin con respecto a esta división y creación del mundo: "Su naturaleza original se conservó en la parte inferior del cosmos; la parte superior, en cambio, adquirió características masculinas. La separación de ambas partes de la diosa fue mantenida con postes -mencionados como árboles, hombres, dioses...- que impidieron la recomposición de la diosa" (López-Austin, 18). Dos de estos dioses, Quetzalcoatl y Huitzilopochtli crearon a Cipactli, una especie de caimán sobre el cual hicieron la tierra. Continúa explicando López-Austin:

Para una profundización del concepto de ollin y nepantla, véase W. Elzey "Some remarks on the space and time of the 'Center' in Aztec religion". 
Una vez creados la tierra y el cielo a partir del cuerpo de Cipactli, y con ello constituida la gran división entre la parte femenina y la masculina del cosmos, los cuatro postes -o árboles o dioses u hombres- se convirtieron en los caminos de los dioses. Fueron los caminos de los dioses porque por su tronco hueco correrían y se encontrarían las esencias divinas opuestas que eran los flujos de las dos mitades del cuerpo del monstruo. (López-Austin, 19).

Observando la división cuatripartita del mundo en el Códice FejérváryMayer, podemos entender análogamente estos cuatro postes que soportan la división del mundo. La energía divina que estos cuatro dioses expulsan luego de su sacrificio se convertirá, entonces, en las almas de los hombres, animales, piedras, montes, agua, etc. Así, como argumenta López-Austin: "Los dioses actúan cíclicamente en el mundo. La sustancia divina es enviada a la tierra en forma de tiempo, de destino. Esto hizo que el calendario mostrara las combinaciones de las distintas fuerzas de los dioses. Todos ellos entraban en el juego de jerarquías y dominios" (López-Austin, 1994: 28). En otras palabras, la organización del mundo se da en la interacción y alternancia de estos cuatro dioses en los cuatro costados del mundo, para dotar a éste y a sus habitantes del tonalli, la energía divina para dar movimiento al mundo y al hombre. La narración de López-Austin, en este sentido, alegoriza de otro modo la historia del Quinto Sol en cuanto a que expresa la idea de conjunción de estas fuerzas, el sacrificio de los dioses y, por lo tanto, su entrega de la energía-movimiento para el Sol y desenvolvimiento del complejo cahuitl/ tlacauhtli.

\section{El altepetl y la organización del territorio}

Nuestro objetivo, ahora, es ver hasta qué punto la organización del espacio cosmológico que encierra el concepto de tlacauhtli determina otro tipo de organización espacial usada por los nahuas a finales del siglo XV y principios del XVI, y que conocemos como altepetl (pl. altepeme). Construida en base al difrasismo in alt, in tepetl, que quiere decir "las aguas, las montañas", altepet/ refiere a un territorio en particular y una organización de personas ejerciendo dominio sobre éste. De acuerdo a James Lockhart, los altepetl son la unidad básica de organización sociopolítica que recibe y administra el tributo de la gente que reside en él. Más allá de la historia de diferentes alianzas interétnicas que configuraron el panorama del valle central de México, es el altepetl la estructura que se mantuvo incluso después de la llegada de Hernán Cortés. Esto se explica ya que los nahuas lo diseñaron de acuerdo a entidades relativamente iguales, separadas y simétricas, elementos que pudieron perdurar por mucho tiempo incluso tomando en cuenta la transformación del altepetl a "pueblo" español con 
su configuración de sujeto-cabecera. Un altepetl, entonces, se enmarca dentro de un territorio, posee una figura que cumple una función de administrador llamado tlatoani ("el que habla", pl. tlatoque), quien proviene de una dinastía de alguna de las élites del altepetl. El grupo humano que lo conforma posee una historia de migraciones que explican su asentamiento final en un territorio determinado; $y$, finalmente, posee estructuras arquitectónicas importantes para el desenvolvimiento de la vida social como lo son el templo dedicado al dios tutelar (teocalli), un palacio para el tlatoani, literalmente "quien habla", lugar llamado (tecpan); y, un mercado para las transacciones de alimentos y ropas (tianquiztli). Según explica Lockhart, la manera que tenían los antiguos nahuas de organizar las estructuras más grandes en todo ámbito (político, económico, artístico, etc.) fue creando pequeñas partes constitutivas del todo, las que debían ser relativamente iguales y separadas. La unidad de estas partes consistía en una ordenación simétrico-numérica de éstas a partir de una rotación cíclica. Estas partes, finalmente, funcionaban de una manera celular o modular, en donde la jerarquía solo se establecía para la rotación de estas partes (Lockhart, 1992: 15). De aquí a que Bernardo García Martínez señale que el altepetl no se establece de una manera rígidamente centralizada (1987: 76). Ahora bien, del altepetl queremos destacar la organización del grupo comunitario en el territorio y ver posibles conexiones con los principios de movimiento y centralización. Para ello, deberemos analizar las entidades constitutivas del altepetl llamadas calpolli o tlaxilacalli ${ }^{3}$.

Los calpolli conformaban un grupo étnico dentro del altepetl y se establecían en un número fijo par que, generalmente, era de cuatro, seis, ocho o, excepcionalmente, siete. El número par destacaba la relación simétrica entre los calpolli, siendo el conjunto de cuatro la constitución ideal tomando en cuenta las direcciones cardinales que ya mencionamos más arriba, aunque también aparecen grupos de seis, ocho (duplicación de cuatro) y siete, caso excepcional que puede encontrar justificación en la historia de los siete linajes venidos de Chicomoztoc. Cada calpolli tenía un nombre en particular, el cual hacia referencia al origen étnico del grupo o a la localización geográfica de éste. Al mismo tiempo, existía un jefe llamado teuctlatoani, quien desempeñaba la tarea de administrador del tributo y organización del grupo al mismo tiempo que lo representaba en los consejos del altepetl. Por último, sabemos que constaban de veinte, cuarenta, ochenta o hasta incluso cien familias. Ahora bien, cada calpolli

3 De acuerdo a James Lockhart, algunos textos ocupan el término calpolli para la fase de migración hacia el territorio final de los grupos que conformaban el altepetl; en cambio, tlaxilacalli se ocupaba para los grupos ya cuando estos se encontraban en un territorio fijo. Debido a la extensión del uso calpolli en los estudios mesoamericanos, usaremos éste de aquí en adelante. 
interactúa dentro del altepet/ de una manera rotativa y cíclica a semejanza de la estructura del espacio cosmológico. Si en este último tenemos a un grupo de dioses buscando equilibrio en el mundo en la repetitiva alternación de sus poderes para crear la Quinta Edad, el calpolli debe hacer lo mismo dentro del altepetl. Para ello, debe prestar servicios al ente global de una manera rotativa con los otros grupos. Por ejemplo, entrega una parte de su maíz y otros productos al tlatoani alternadamente con los otros grupos. Además, aporta hombres para el trabajo de tierras, para el funcionamiento del templo, o para la guerra, de manera rotativa. El establecimiento del orden viene dado de distintos factores. Algunas veces, la situación geográfica del grupo otorga los turnos en donde la cuenta comienza por el que se encuentra en el oriente. En su mayoría, la rotación se ordena por la antigüedad del calpolli, siendo los primeros grupos migratorios los que comienzan con el turno. Tal como señala Lockhart, el orden rotativo de los calpolli fue el alma del altepetl (1992: 17).

Figura 2 Organización celular de los calpolli en un altepetl hipotético

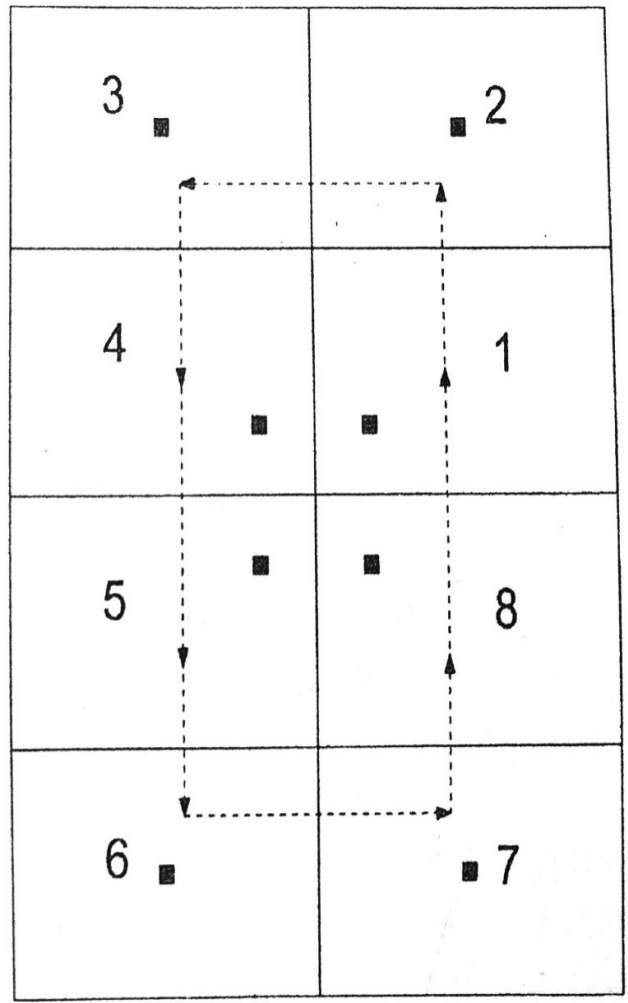

Fuente: Lockhart (1992) 
Figura 3 Distribución de ocho calpolli, según Van Zantwijk

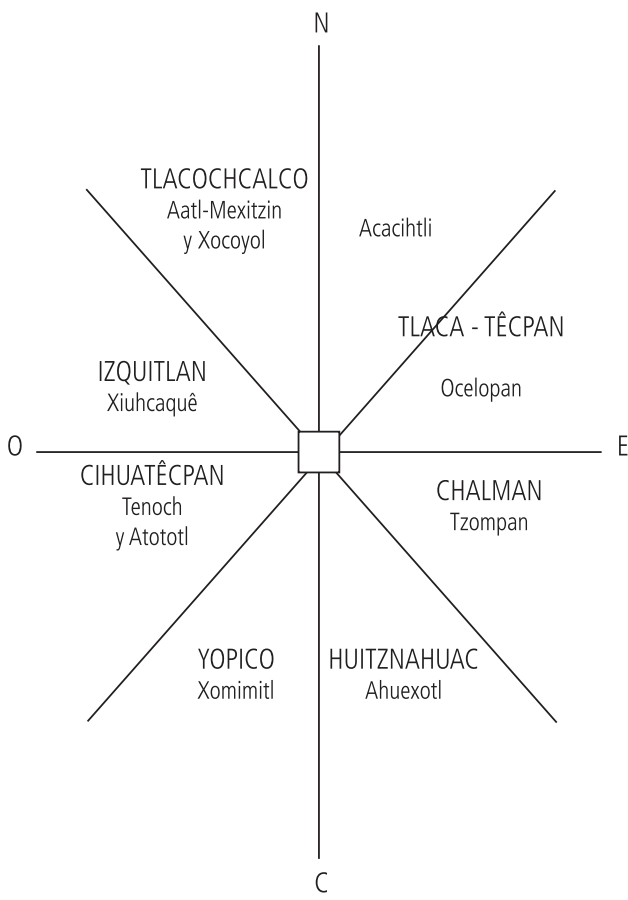

Fuente: Fernández y García (2006)

Como mencionamos anteriormente, este tipo de organización cíclica probablemente basado en la estructuración del espacio cosmológico, se establecía a distintos niveles. De esta manera, por ejemplo, un grupo de altepeme se organizaba en una estructura idéntica. Por ejemplo, Tenochtitlan funcionaba con cuatro altepetl como se muestra en la figura 4 (en orden: Moyotlan, Teopan, Atzaqualco o Tzaqualco, y Cuepopan). A diferencia de la estructura más simple, en esta conformación la administración no recaía en una sola persona sino que la conformaban los tlatoques de los otros altepeme, quienes debían elegir como representante ceremonial a quien provenía del más antiguo altepetl. Más allá de esto, la relación del altepetl con los otros altepeme funcionaba de la misma manera que el calpolli con el altepetl en cuanto a tributos, trabajo, etc., distribuyendo los turnos rotativamente por la antigüedad. Por lo tanto, podemos ver que la idea de movimiento y circularidad, tanto en la organización del espacio como en la del territorio, se establecen como el motor de la interacción. Este movimiento es alternado y cíclico, como los ca/polli que dan vida al altepetl, pero también como los dioses que sostienen al Quinto Sol. No es menor, por ende, que durante la ceremonia del Fuego Nuevo cada cincuenta y dos años $(13 \times 4=52)$, se llevara fuego desde las cuatro partes del territorio 
dominado por la Triple Alianza. Por último, cabe preguntarse si es que la mínima estructura espacial, la tierra o, como se denomina en náhuatl, tlalli, posee en su concepción la idea de movimiento, permitiéndonos conectar los distintos niveles del espacio.

Figura 4 Organización de Tenochtitlan

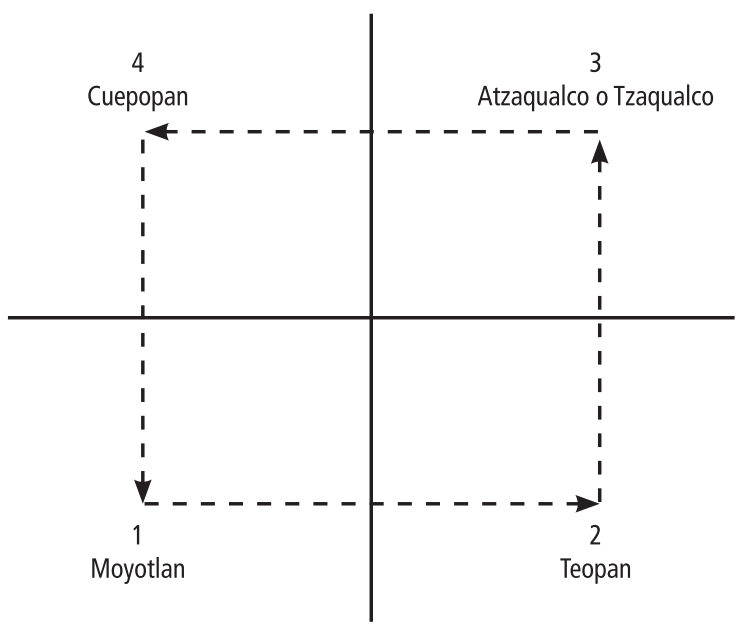

Fuente: Lockhart (1992)

\section{Tlalli y la circulación del tributo}

Los antiguos nahuas identificaban el concepto "tierra", así como el "planeta Tierra", pero también como "la tierra en donde estoy parado", con la palabra tlalli. Sin embargo, el término se expande para englobar la idea de "heredad". De esto se desprende que este concepto espacial tenga alcances de tipo económico relacionados no solo con la "propiedad" de la tierra sino que, más importante aún, con el producto que de ella se obtiene. De aquí que los nahuas configuraran dos tipos de clasificaciones de la tierra. Por un lado, una clasificación de acuerdo a quién es dueño del producto de esta tierra y, por otro, cuán productiva es ésta. Comencemos por esta última para luego observar la relación entre propiedad, producto y circulación del tributo.

En su libro Estructura Económica de la Sociedad Mexica, Víctor Castillo nos provee de nueve categorías de tlalli de acuerdo a su estado productivo: 1) Atloctli, tierra buena, blanda, húmeda y creadora de cosas; 2) Cuauhtlalli, tierra fructífera, de árboles podridos u hojarasca, tierra áspera, obscura o quizás amarilla; 3) Tlalcoztli, tierra fértil, amarilla, hacedora de cosas; 4) Tlalhuitectli, tierra arada, compuesta, relabrada; 5) Tlalahuiac, tierra que ha sido 
bien cuidada, que se abona; 6) Atlalli, tierra regada y buena para sembrar frijol; 7) Tepetlalli, cuerpo del cerro, en lo alto, en la cuesta, es tierra seca, de barro duro, tierra ceniza; 8) Tetlalli, tierra que está en los cerros, pedregosa, áspera, seca, y es allí donde nace el maíz duro, aunque irrigada produce cosas; y 9) Tlalzolli, tierra vieja o en barbecho. Como podemos apreciar en esta clasificación, la tierra se concibe como sustento y como medio de producción. De aquí a que Pedro Carrasco reconozca que tanto la tierra como el trabajo sean los elementos esenciales de la economía nahua previo a la llegada de Cortés. En el caso mexica, el control del tlalli o, mejor dicho, de la economía, está fuertemente regulado por un mecanismo político:

"La base de la economía era una estructura de dominación definida por la existencia de dos estamentos fundamentales: los nobles (pipiltin), que formaban, como personal de gobierno, la clase dominante que controlaba los medios materiales de producción, y los plebeyos (macehualtin), que eran la clase trabajadora dependiente, política y económicamente, de la nobleza" (Carrasco, 1978: 23-24).

Como explica Carrasco, es el altepetl, por medio de la clase dirigente, el que designa las tierras que serán trabajadas. Para ello, existía una clasificación de cuál territorio se le asignaría para obtener el usufructo de la tierra. De esta manera, el tlalli se divide en calpullalli, tierras poseídas en forma comunal por los integrantes de cada calpulli; y altepetlalli, tierras de la ciudad. Las calpullalli eran tierras cultivadas comunalmente por los macehuales para cubrir el pago de los tributos del calpulli. Los miembros del calpulli podían disfrutar la tierra de por vida bajo las restricciones de no tener derecho a enajenarla, ni dejar de labrarla durante un período máximo de tres años, si no se perdía (Castillo, 1984: 77). Las altepetlalli eran las tierras de la ciudad y se dividían en: a) teopantlalli, tierras de los templos, las cuales eran trabajadas por los macehuales para sustentar los gastos del cuerpo sacerdotal y las celebraciones religiosas; b) tlatocatlalli o tlatocamilli, tierras del señorío que se arrendaban para cubrir los gastos del palacio y que se asignaban para el usufructo del tlatoani; c) tecpantlalli, tierras cuyos frutos sirven para el mantenimiento de los servidores del palacio; d) tierras de los tecuhtlatoque (jueces), cuyos usufructos sirven para pagar los servicios de aquellos; e) milchimalli y cacolomilli, tierras para aprovisionar a los guerreros durante las batallas; y, f) yastlalli, las tierras que eran tomadas de los enemigos como botines de guerra. Además, estaban las pillalli, las tierras de los pipiltin o nobles, quienes poseían los derechos de estas tierras o los heredaban, y tecpillalli que eran tierras de los de ilustre cepa (tecpiltin). Como se puede observar, las tierras eran asignadas con respecto a la función que cumplía el sujeto dentro de la sociedad mexica. No había manera de comprar ni vender la tierra ya que no eran dueños de ellas sino que de sus usufructos. En relación con esto, Carrasco argumenta que los nobles que gozaban de las tierras asignadas tenían la obligación de servir 
en distintos puestos, siendo los bienes que otorgaban estas tierras lo que les permitía dedicarse de lleno a esas actividades (Carrasco, 1978: 25). Ahora bien, el macehual también obtenía cosas a cambio: "... se beneficiaba de las obras públicas y de las redistribuciones de bienes; recibía tierra para que sustentara, protección en caso de conflicto y los beneficios del culto público organizado por los señores" (Carrasco, 1978: 45).

Si el tlalli se entiende solo en conexión con el tributo que genera, por lo tanto, su determinación espacial se asigna en cuanto al sistema tributario. En este caso, la circulación del tributo en el México prehispánico viene dado, según explica Johanna Broda, por una división en cinco grandes regiones correspondientes a los rumbos centro-norte-oeste-sur-este ${ }^{4}$. Esta circulación, sin embargo, no se establece de una manera cíclica o rotativa como la organización del espacio cosmológico o las diferentes entidades del altepetl, sino que, como lo entiende Frances Berdan, en un movimiento unidireccional del macehualli provincial a los miembros de la nobleza azteca ${ }^{5}$ (Berdan, 1978: 187). En este sentido, el tlalli es un espacio en movimiento ya que su producto es el que viaja al centro y se vuelve a distribuir donde lo requiera la clase dirigente. En sí, el tlalli también es cíclico y temporal de acuerdo a la producción y la estación del año, pero es movimiento en cuanto a que conlleva una dirección redistributiva dentro de las relaciones sociales entre macehualli, el que trabaja la tierra, y pipiltin, quien posee el usufructo de ésta.

\section{Reflexiones finales}

Los conceptos de tlacauhtli, altepetl y tlalli responden a tres dimensiones de la expresión espacial mesoamericana. Como hemos podido observar, estas dimensiones se encuentran íntimamente conectadas por la idea de movimiento y centralidad. En el caso de tlacauhtli, lo que vendría siendo la idea cosmológica de espacio entre los nahuas, vemos la idea de movimiento y temporalidad en su definición gracias a los relatos míticos del Quinto Sol. Por medio de la idea de armonización y equilibrio entre los cuatro puntos cardinales del espacio identificados con cuatro dioses y cuatro épocas previas, el espacio aparece en la existencia nahua. El movimiento de este espacio cosmológico viene dado por la alternancia de sus cuatro pilares en la influencia divina en el complejo tiempo/espacio y en el devenir de los

4 Véase el artículo de Broda "El tributo en trajes guerreros y la estructura del sistema tributario mexica" en Economía Política e Ideología en el México Prehispánico.

5 Para un análisis más detallado de las relaciones recíprocas y redistributivas en el México antiguo, véase el capítulo de Frances Berdan en Economía Política e Ideología en el México Prehispánico, titulado "Replicación de principios de intercambio en la sociedad mexica: de la economía a la religión". 
hombres. Tal alternancia y movimiento de sus fuerzas es replicado en la organización política del altepetl, el cual se estructura por la rotación de sus partes constitutivas, llamadas calpolli, para la subsistencia del ente macro mediante tributos y trabajo. Derivado de esto, el tlalli, el cual pertenece al estado en todas sus facetas, se organiza en base a su producción y a su asignación a la clase social. Esta organización se constituye para distribuir los bienes que otorga el t/alli, otorgándole un dinamismo y movimiento a la tierra y sus productos.

\section{Referencias bibliográficas}

CARRASCO PIZANA, P.; J. Broda, e Instituto Nacional de Antropología e Historia (México). Centro de Investigaciones Superiores (1978). Economía Política e Ideología en el México Prehispánico. 1. ed. México. D.F.: Editorial Nueva Imagen.

CASTILLO, V. (1984). Estructura económica de la sociedad Mexica. México, D.F.: UNAM.

ELZEY, W. (1976). "The Nahua Myth of the Suns: History and cosmology in PreHispanic Mexican Religious", en Numen, 23, 2.

CASTILLO, V. (1976). "Some remarks on the space and time of the "Center" in Aztec religion", en Estudios de Cultura Náhuatl, 12.

FERNÁNDEZ CHRISTLIEB, F. y A. García Zambrano (2006). Territorialidad y paisaje en el Altepetl del siglo XVI. Sección de Obras de Historia. 1. ed. Mexico, D.F.: Fondo de Cultura Económica: Instituto de Geografía de la Universidad Nacional Autónoma de México.

FLORESCANO, E. y R. Velázquez (2002). Memoria mexicana. Sección de Obras de Historia. 3. ed. México: Fondo de Cultura Económica.

GARCÍA MARTínEZ, B. (1987). Los pueblos de la sierra: El poder y el espacio entre los indios del norte de Puebla hasta 1700. 1a ed. México, D.F.: El Colegio de México, Centro de Estudios Históricos.

GIBSON, C. (1964). The Aztecs under spanish rule; a history of the indians of the Valley of Mexico, 1519-1810. California: Stanford University Press.

JANSEN, M. E. R. G. N., et al. (1994). Códice Fejérváry-Mayer. Códices Mexicanos. 1. ed. Austria México: Akademische Druck- und Verlagsanstalt; Fondo de Cultura Económica.

LEÓN-PORTILLA, M. (1966). La filosofía Náhuatl estudiada en sus fuentes. Universidad Nacional Autónoma de México; Instituto de Investigaciones Históricas. Serie de Cultura Náhuatl, Monografías, 3. ed. México: Universidad Nacional Autónoma de México, Instituto de Investigaciones Históricas. 
LEÓN-PORTILLA, M. (2003). Toltecáyotl: Aspectos de la cultura Náhuatl. México, DF: Fondo de Cultura Económica.

LOCKHART, J. (1992). The Nahuas after the conquest: A social and cultural history of the indians of Central Mexico, Sixteenth through Eighteenth Centuries. California: Stanford University Press.

LÓPEZ-AUSTIN, A. (1994). Tamoanchan y Tlalocan. Sección de obras de Antropología. 1. ed. México: Fondo de Cultura Económica.

MOLINA, A. (1970). Vocabulario en lengua castellana y mexicana y mexicana y castellana. Biblioteca Porrúa. México, D.F.: Editorial Porrúa.

SOUSTELLE, J. (1996). El universo de los Aztecas. México, D.F.: Fondo de Cultura Económica. 